\title{
Morphology of the Surface of Technically Pure Titanium VT1-0 after Electroexplosive Carbonization with a Weighed Zirconium Oxide Powder Sample and Electron Beam Treatment
}

\author{
Kirill V. Sosnin ${ }^{1}$, Sergey V. Raykov, Ekaterina S. Vaschuk ${ }^{1}$, \\ Yuri F. Ivanov ${ }^{2,3, a)}$, Evgenie A. Budovskikh ${ }^{1}$, and Victor E. Gromov ${ }^{1, b)}$ \\ ${ }^{1}$ Siberian State Industrial University, Novokuznetsk, 654007, Russia \\ ${ }^{2}$ Institute of High-Current Electronics SB RAS, Tomsk, 634055, Russia \\ ${ }^{3}$ National Research Tomsk Polytechnic University, Tomsk, 634050, Russia \\ a)yufi55@mail.ru \\ b) Corresponding author: gromov@physics.sibsiu.ru
}

\begin{abstract}
Titanium is carbonized by the electroexplosive method. Formation of a surface alloyed layer and a coating on the treated surface is established by the methods of transmission electron microscopy. The morphology and elemental composition of the alloyed layer are analyzed. A dependence of the structure of the modified layer subjected to electron gun treatment on the absorbed power density is revealed.
\end{abstract}

Keywords: titanium, electroexplosive alloying, carbonization, zirconium oxide, electron gun treatment, scanning electronic microscopy, structurally-phase states

\section{INTRODUCTION}

One of the most promising practical applications of electroexplosive alloying is the development of a method for combined hardening of the surface of metals and alloys, including electroexplosive alloying by condensed particles of explosion products and subsequent treatment of the alloyed surface by high-intensity electron beams $[1,2]$. The formation of nonequilibrium structurally-phase states in the surface layer subjected to electron beam treatment during micro- and submillisecond time intervals is determined by ultrahigh heating rates (up to $10^{6} \mathrm{~K} / \mathrm{s}$ ) of a thin surface layer of the material $\left(10^{-4}-10^{-3} \mathrm{~mm}\right)$ to supercritical temperatures and formation of limiting temperature gradients (to $10^{7}-10^{8} \mathrm{~K} / \mathrm{m}$ ) providing cooling of the surface layer due to heat transfer to the bulk of the material with a rate of $10^{4}-10^{9} \mathrm{~K} / \mathrm{s}$. Thus, the application of pulsed low-energy high-current electron beams for modification of the surface layers through the formation in them of nonequilibrium structurally-phase states opens new possibilities for the development of highly efficient technologies of hardening of metals and alloys of different designations. The present work is aimed at investigation of the structure and phase composition of the surface layer of technically pure titanium samples subjected to electroexplosive carbonization together with zirconium dioxide powder and subsequent electron beam treatment.

\section{MATERIAL AND INVESTIGATION TECHNIQUE}

We investigated samples of technically pure titanium VT1-0 subjected to electroexplosive alloying on the EVU 60/10 electroexplosive system [3]. Carbon fibers (CF) of the LU-P/0.1-50 carbon ribbon (LLC "Argon", Balakovo) were used as a conductive materials. The zirconium dioxide powder $\mathrm{ZrO}_{2}$ was placed on the carbon fibers surface in

International Conference on Physical Mesomechanics of Multilevel Systems 2014 AIP Conf. Proc. 1623, 603-606 (2014); doi: 10.1063/1.4899017

(C) 2014 AIP Publishing LLC 978-0-7354-1260-6/\$30.00 
the explosion region. The mass of the explosive carbon fibers ribbons was $70 \mathrm{mg}$, the mass of the weighed powder sample was $50 \mathrm{mg}$, and the power density absorbed during treatment was $5.5 \mathrm{GW} / \mathrm{m} 2$. Some samples were further treated by an electron beam on the "SOLO" setup developed at the High-Current Electronics Institute of the Siberian Branch of the Russian Academy of Sciences $[1,2]$. The parameters of treatment were the following: electron energy of $18 \mathrm{keV}$, electron beam pulse repetition frequency of $0.3 \mathrm{~Hz}$, electron beam energy density varied from 45 to $60 \mathrm{~J} / \mathrm{cm}^{2}$, acting pulse duration changed from 100 to $200 \mu \mathrm{s}$, and the number of acting pulses changed from 10 to 20 . The chosen ranges of the irradiation parameters allowed the surface layer to be modified in the regime of high-speed melting. The morphology and elemental composition of the treated surface and its transverse microsections were investigated by the methods of scanning electron microscopy (with a LEO EVO 50 scanning electron microscope).

\section{RESULTS OF INVESTIGATIONS AND DISCUSSION}

We have already demonstrated that products of electric explosion of a conductor represent a multiphase system including both the plasma component and the condensed particles of various sizes. In the process of jet formation, its front forms the plasma component while the more inertial condensed particles are located in the rare part of the jet. This causes not only surface alloying, but also formation of the coating, as a rule, very porous and containing considerable amount of the drop fraction, microcraters and microcracks that significantly decrease specifications of the treated samples. The characteristic image of the morphology of the coating formed on the surface of samples of technically pure titanium alloyed by electric explosion of carbon fibers with a weighed zirconium dioxide powder sample is shown in Fig. 1.

The coating was characterized by high level of roughness and wide variety of structural elements - micropores, microcraters, microcracks, splashes caused by radial metal flow, layers formed as a result of metal splashing when powder or carbon fibers fall into the melt, volumes formed by highly dispersed round formations (particles), rods of various sizes, formations with cellular structure (with cell sizes changing from 80 to $300 \mathrm{~nm}$ ) - revealed by the methods of metallography and scanning electron microscopy.

The coating formed on the sample surface was characterized by a very inhomogeneous distribution of the alloying elements (carbon and zirconium). An analysis of the surface structure by the methods of scanning electron microscopy in backscattered electrons revealed surface regions that differed by their contrasts (Fig. 2). On the image of the surface light regions were clearly seen whose sizes changed from 5 to $30 \mu \mathrm{m}$ (Fig. 2(a)) and dark colored rods from 10 to $50 \mu \mathrm{m}$ long and from 2 to $4 \mu \mathrm{m}$ thick (Fig. 2(b)).

In backscattered electron images, regions of the material enriched by atoms having greater atomic weight are light; on the contrary, regions of the surface enriched by atoms having relatively small atomic weight are dark. In our case, zirconium possesses the greatest atomic weight; hence, globular regions are enriched by zirconium atoms. Carbon has relatively small atomic weight; hence, the rod-like formations are fragments of carbon fiber.
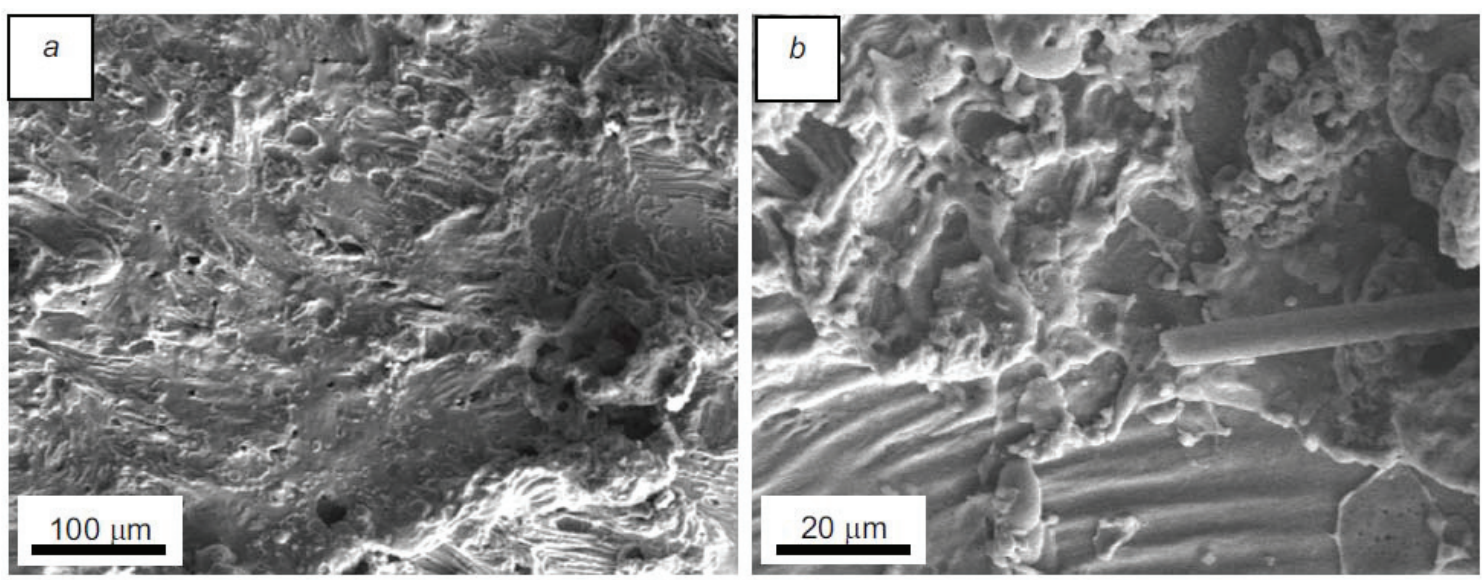

FIGURE 1. Morphology of the surface of technically pure titanium VT1-0. Alloying by electric explosion of carbon fiber with weighed zirconium oxide powder sample. Scanning electron microscopy 

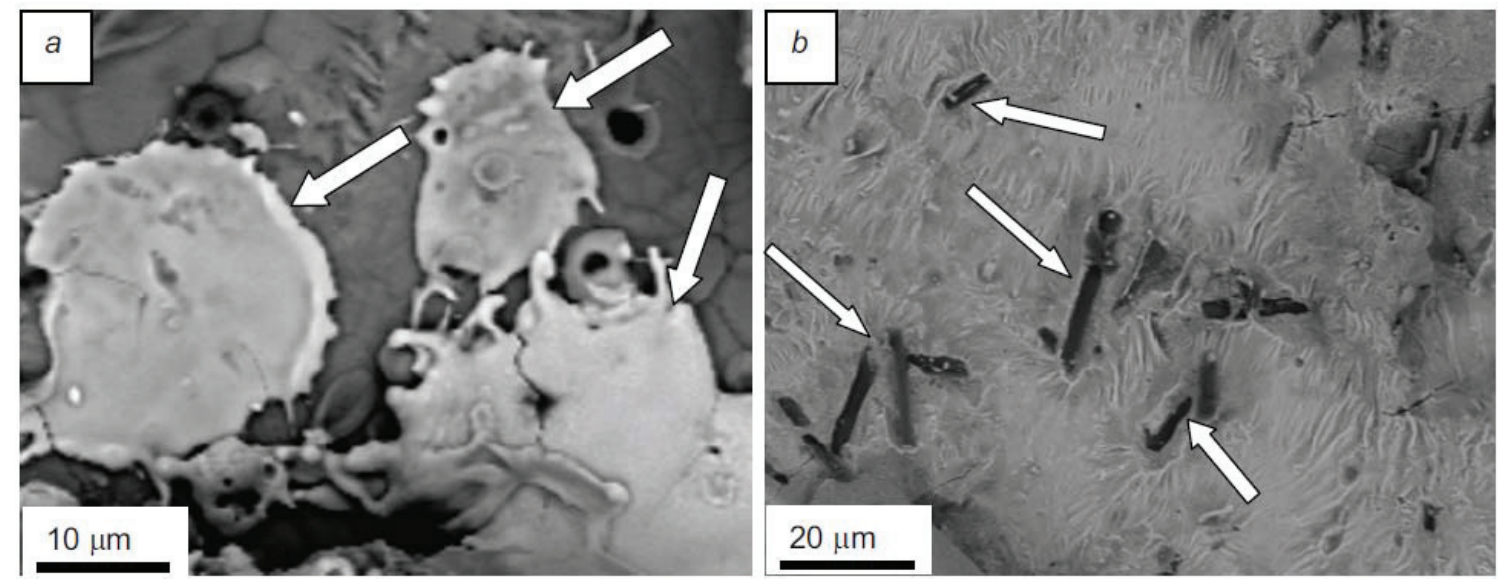

FIGURE 2. Structure of the surface of technically pure titanium VT1-0. Alloying by electric explosion of carbon fiber with a weighed zirconium oxide powder sample. Scanning electron microscopy. Arrows indicate regions enriched by zirconium (a) and carbon (b)

The X-ray spectral microanalysis of the titanium surface after electroexplosive alloying performed by the methods of scanning electron microscopy confirmed the data presented above on the inhomogeneous zirconium distribution in the surface layer structure. In the spectrum of characteristic X-ray radiation recorded in the region of the surface layer possessing light contrast, an analysis of the backscattered electron images demonstrated that radiation of zirconium atoms prevailed (Fig. 3(a)); on the contrary, in the spectrum of characteristic X-ray radiation of the surface region that had no light regions, radiation of titanium atoms prevailed (Fig. 3(b)).

Thus, electroexplosive alloying of titanium was accompanied by the formation of the surface layer characterized by the developed relief and very inhomogeneous distribution of alloying elements. As a rule, the coating formed after electroexplosive alloying was removed by additional grinding and polishing of the surface, which led to partial losses of the alloying elements and reduction of the thickness of the hardened zone. In the present work, the surface of the material subjected to electroexplosive alloying was irradiated by a high-intensity electron beam modifying the structure of the surface and subsurface layers by their re-melting with subsequent high-rate cooling due to heat transfer to the bulk of the sample.

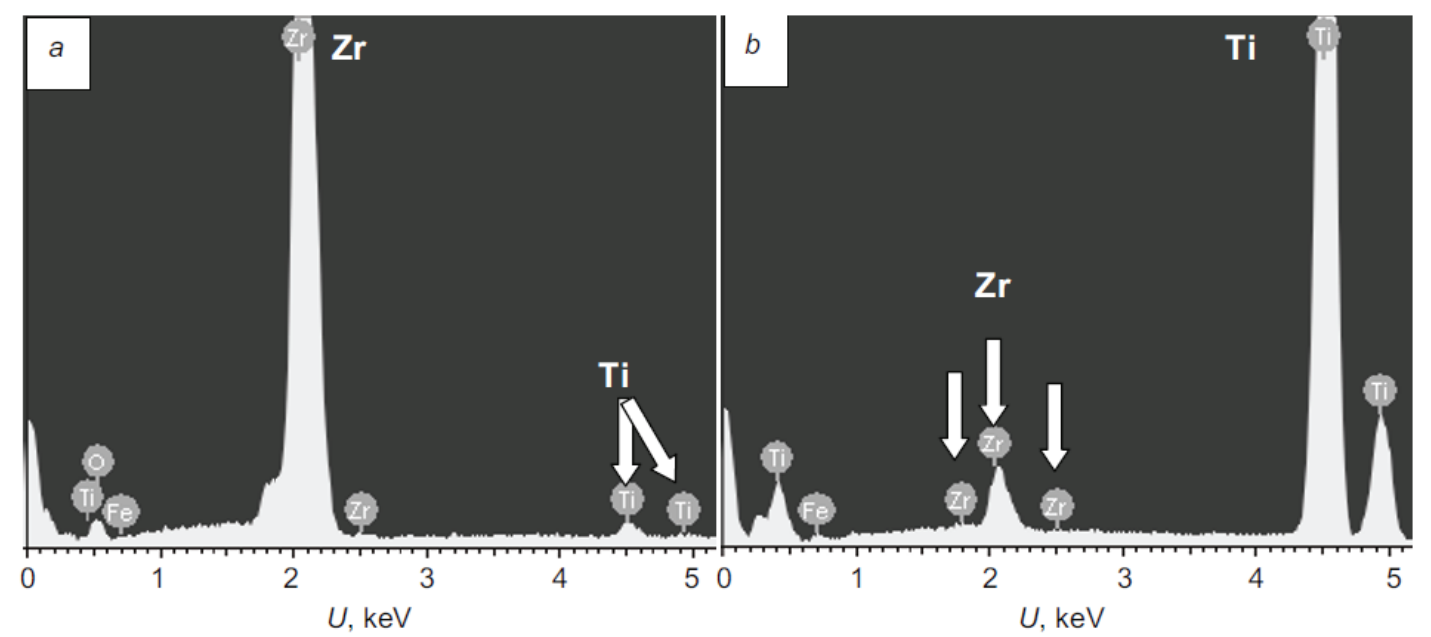

FIGURE 3. X-ray spectra of the surface layer of technically pure titanium subjected to electroexplosive alloying. Here (a) shows spectra corresponding to the light regions designated by arrows in Fig. 2(a) and (b) shows spectra of the regions having grey contrast 


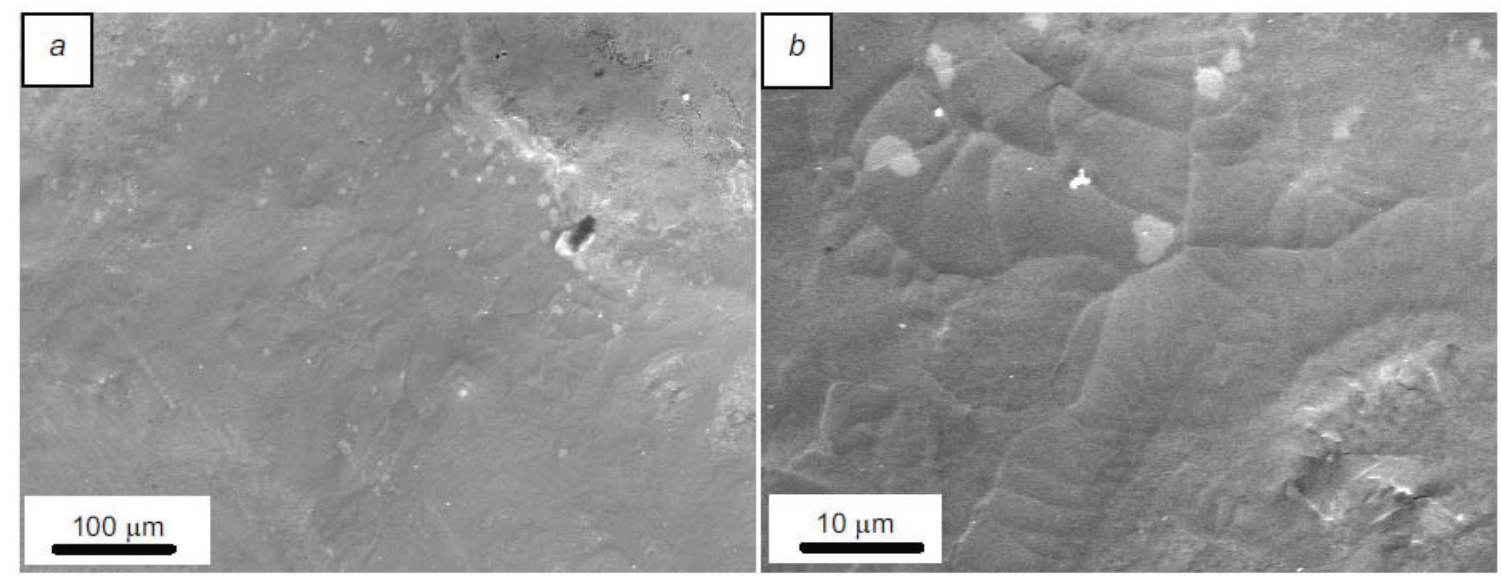

FIGURE 4. Structure of the surface after electroexplosive alloying of technically pure titanium VT1-0 subjected to electron beam treatment $\left(45 \mathrm{~J} / \mathrm{cm}^{2}, 100 \mu \mathrm{s}, 10\right.$ pulses, and $\left.0.3 \mathrm{~Hz}\right)$. Scanning electron microscopy

Investigations of the irradiated surface by the methods of scanning electron microscopy demonstrated that after surface treatment with an electron beam having electron energy density of $45 \mathrm{~J} / \mathrm{cm}^{2}(100 \mu \mathrm{s}, 10$ pulses, and $0.3 \mathrm{~Hz})$, the sample surface radically changed. In the central part of the region of interaction with the electron beam $(\sim 10 \mathrm{~mm}$ in diameter) microdrops, microcraters, and microcracks disappeared, and the surface relief became smooth (Fig. 4(a)). The polycrystalline structure with grain sizes of 5-15 $\mu \mathrm{m}$ was formed (Fig. 4(b)).

\section{SUMMARY}

The surface of technically pure titanium VT1-0 was alloyed by electric explosion of carbon fibers with weighed zirconium oxide powder sample. The surface after electroexplosive alloying was treated in the regime of melting by electron beam. The morphology of the alloyed surface, the phase, and the elemental composition of the surface layer were investigated. Regimes of irradiation of the surface coating by a high-intensity pulsed electron beam were established that allowed dense extended surface layers to be formed smoothly transforming into the structure of the main alloy.

This work was supported in part by the Russian Foundation for Basic Research (Scientific Project No. 13-0212009 ofi_m) and by the Ministry of Education and Science of the Russian Federation State Assignment 2708 G3.

\section{REFERENCES}

1. E. A. Budovskikh, S. V. Karpij, and V. E. Gromov, Bull. Russ. Acad. Sci. Phys. 73(9), 1253 (2009).

2. S. V. Karpii, M. M. Morozov, Yu. F. Ivanov, et al., Steel Transl. 40(8), 723 (2010).

3. D. A. Rovanov, E. A. Budovskikh, Y. D. Zhmakin, and V. E. Gromov, Steel Transl. 41(6), 469 (2011). 